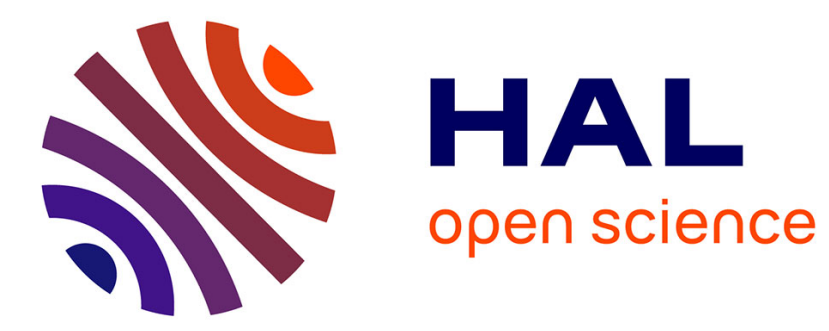

\title{
Delayed electron emission from fullerene dianions: Evidence for a thermal tunneling detachment
}

\author{
Bruno Concina, Franck Lepine, C. Bordas
}

\section{To cite this version:}

Bruno Concina, Franck Lepine, C. Bordas. Delayed electron emission from fullerene dianions: Evidence for a thermal tunneling detachment. Physical Review A, 2015, 92, pp.023410. 10.1103/PhysRevA.92.023410 . hal-02308163

\section{HAL Id: hal-02308163 https://univ-lyon1.hal.science/hal-02308163}

Submitted on 12 Feb 2021

HAL is a multi-disciplinary open access archive for the deposit and dissemination of scientific research documents, whether they are published or not. The documents may come from teaching and research institutions in France or abroad, or from public or private research centers.
L'archive ouverte pluridisciplinaire $\mathbf{H A L}$, est destinée au dépôt et à la diffusion de documents scientifiques de niveau recherche, publiés ou non, émanant des établissements d'enseignement et de recherche français ou étrangers, des laboratoires publics ou privés. 


\title{
Delayed electron emission from fullerene dianions: Evidence for a thermal tunneling detachment
}

\author{
Bruno Concina, ${ }^{*}$ Franck Lépine, and Christian Bordas \\ Institut Lumière Matière, UMR5306 Université Claude Bernard Lyon 1-CNRS, Université de Lyon, 69622 Villeurbanne cedex, France
}

(Received 9 February 2015; revised manuscript received 15 June 2015; published 11 August 2015)

\begin{abstract}
Isolated multiply charged anions exhibit a repulsive Coulomb barrier (RCB) towards electron loss. In this paper, the role of tunneling through the RCB in the delayed electron emission is addressed. Electron kinetic energy distributions of photoexcited fullerene dianions $\mathrm{C}_{84}{ }^{2-}$ and $\mathrm{C}_{90}{ }^{2-}$ have been measured with a velocity-map imaging spectrometer for delays of about $150 \mathrm{~ns}$ after the nanosecond laser excitation. On such spectra, delayed electron emission is unambiguously separated from direct detachment. These measurements display significant delayed emission below the RCB. A detailed-balance model including electron tunneling through the RCB has been developed. The very good agreement between the measurements and the model provides quantitative evidence for a thermal tunneling electron emission.
\end{abstract}

DOI: 10.1103/PhysRevA.92.023410

PACS number(s): 33.80.Eh, 36.40.Qv

Isolated multiply charged anions (MCAs) have attracted significant interest because of the very specific interaction experienced by the outer electron. Whereas in other molecular systems (neutrals, cations, and singly charged anions), the outer electron interacts with the core via an attractive potential, MCAs are characterized by an asymptotic repulsive interaction. The combination of the long-range Coulomb repulsion and the short-range attractive interactions results in the formation of a repulsive Coulomb barrier (RCB) as depicted in Fig. 1 for $\mathrm{C}_{84}{ }^{2-}$. The existence of the RCB leads to unique features in the photodetachment [1-3].

The direct electron emission has been measured on numerous MCAs with a magnetic bottle apparatus or a velocity-map imaging (VMI) spectrometer [1-3]. In the direct photoelectron spectra, the RCB leads to a cutoff at the low kinetic energy side equal to the barrier height $\varepsilon_{\mathrm{C}}$. The metastable MCAs characterized by a negative binding energy (i.e., a negative second electron affinity $E A_{2}$ ) are revealed by features with kinetic energy larger than the photon energy [1]. The angular distribution measured with a VMI spectrometer is governed by the intramolecular Coulomb repulsion [2]. In addition, adiabatic tunneling has been measured from excited states lying in close proximity to the top of the RCB [4-6]. As a model molecular system, the fullerene dianions have been the subject of several photoelectron spectroscopy studies in which the RCB height $\varepsilon_{\mathrm{C}}$ and the second electron affinity $E A_{2}$ have been deduced from the direct electron emission spectra as a function of the molecular size [7-10].

Another major decay process following photon absorption is thermionic emission: The excitation energy is transferred to the vibrations and leads to a delayed electron emission after thermal equilibration [11]. By contrast with direct emission, very few studies have been devoted to the MCA thermionic emission. Delayed electron emission has been observed on $\mathrm{C}_{84}{ }^{2-}$ using a mass spectrometer [12] and the surviving fraction of $\mathrm{C}_{76}{ }^{2-}$ and $\mathrm{C}_{84}{ }^{2-}$ has been measured in a Penning trap after laser heating $[13,14]$. It must be pointed out that over-the-barrier detachment has been assumed in these contributions (i.e., electron tunneling through the RCB has been

*bruno.concina@univ-lyon1.fr disregarded). Moreover, statistical electron emission has been recently proposed for various resonantly excited dye dianions $[15,16]$.

In this contribution, the kinetic energy release distributions (KERDs) of electrons emitted from the fullerene dianions $\mathrm{C}_{84}{ }^{2-}$ and $\mathrm{C}_{90}{ }^{2-}$ have been measured for delays of about $150 \mathrm{~ns}$ and are therefore unambiguously attributed to a delayed process. They have been compared to a detailed-balance model which includes electron tunneling through the RCB. The very good agreement between experiment and theory provides evidence for a thermal tunneling detachment, a type of electron emission from excited MCA which we quantitatively describe in this contribution.

The experimental setup was previously described [17]. Briefly, the isolated fullerene dianions are produced by electrospray ionization (ESI) [17]. The selection of the ionic species of interest is operated with a mass resolving quadrupole. The ions are perpendicularly accelerated to a linear time of flight (TOF) by a Wiley-McLaren assembly with high voltages $(3.7 \mathrm{kV})$ pulsed at a $350-\mathrm{Hz}$ repetition rate. The linear TOF contains the VMI spectrometer mounted orthogonally to the ion drift tube. An inhomogeneous electric field perpendicular to both the ion trajectory and the focused laser beam accelerates the electrons towards a position-sensitive detector (a microchannel plate detector backed by a phosphor screen). Electron impacts are recorded by a charge coupled device (CCD) camera. The angularly resolved velocity distribution is deduced from the raw image through numerical inversion [18]. It is angularly integrated to obtain the electron KERD. The nanosecond pulsed detachment laser operating at a $350-\mathrm{Hz}$ repetition rate and synchronized with the mass-selected ion bunch is a frequency-doubled diode pumped Nd:YAG laser (532 nm; $2.33 \mathrm{eV}$ photon energy) of $23 \mathrm{~ns}$ pulse duration [full width at half maximum (FWHM)]. At the crossing point with ions, we estimate that the laser beam has a diameter of $0.4 \mathrm{~mm}$ and the fluence is $300 \mathrm{~mJ} / \mathrm{cm}^{2}$, a regime where multiphoton absorption is substantial. The laser polarization is linear and parallel to the position-sensitive detector surface. The time resolution of the VMI is achieved by gating the voltages applied on the position-sensitive detector, the FWHM of the detection efficiency curve being $35 \mathrm{~ns}$. The imaging spectrometer has been calibrated with the spectrum of $\mathrm{I}^{-}$at $349 \mathrm{~nm}$ and a kinetic energy resolution $\Delta \varepsilon$ of $85 \mathrm{meV}$ (FWHM) 


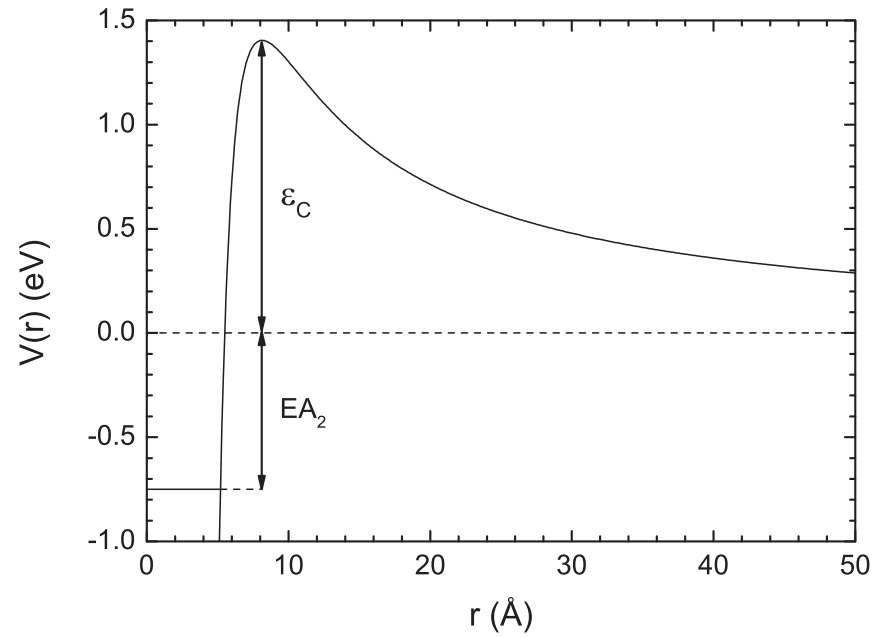

FIG. 1. Interaction potential between the singly charged anion $\mathrm{C}_{84}{ }^{-}$and the electron as a function of the distance $r$ between the anion center and the electron. The zero energy corresponds to an infinite distance. The second electron affinity $E A_{2}$ and the RCB height $\varepsilon_{\mathrm{C}}$ are reported (The electronic ground state is represented as a horizontal line). The potential curve has been calculated assuming that the anion is described as a classical charged dielectric sphere (see text for detail on the model).

has been measured at $\varepsilon=0.494 \mathrm{eV}$. In the present case, the energy resolution is deteriorated by the ion velocity dispersion and by the gating of the detector voltages. The input face of the microchannel plate being connected to the last electrode of the lens arrangement, the fast variation of the detector voltages leads to a slight defocusing of the electron trajectories. Nevertheless, the decrease of the energy resolution has no significant effect on the relatively broad features measured in the delayed electron emission.

In the present experiment, the detection window is delayed by $150 \mathrm{~ns}$ with respect to the laser pulse. Any contribution from direct photodetachment is eliminated for such a delay and thus pure delayed electron emission spectra for a well-defined time window are recorded. The delay must be long enough to avoid any contribution from direct photodetachment and short enough that the dianions are not too far from the center of the VMI spectrometer and the outgoing electrons are detected [which can be estimated by comparing the dianion velocity $(37 \mathrm{~mm} / \mu \mathrm{s})$ and the radius of the inner hole of the VMI electrodes $(10 \mathrm{~mm})]$. A systematic time-resolved study of delayed electron emission is not possible because of the relatively high velocity of the dianions. Nevertheless a single measurement already gives a good insight into the delayed electron emission and imposes stringent constraints to the proposed statistical model.

The KERDs of delayed electron emission from the massselected fullerene dianions $\mathrm{C}_{84}{ }^{2-}$ and $\mathrm{C}_{90}{ }^{2-}$ for a delay of $150 \mathrm{~ns}$ are reported in Fig. 2. They both display two features. The relatively broad feature in the $1 \mathrm{eV}$ range does not display significant differences in both spectra and can be fitted by a Gaussian centered at $1.15 \mathrm{eV}$ of FWHM equal to $0.67 \mathrm{eV}$. The low-energy feature (below $0.5 \mathrm{eV}$ ) is identical to the previously measured KERDs of thermionic emission from the singly charged anions $\mathrm{C}_{84}{ }^{-}$and $\mathrm{C}_{90}{ }^{-}$[17]. Such

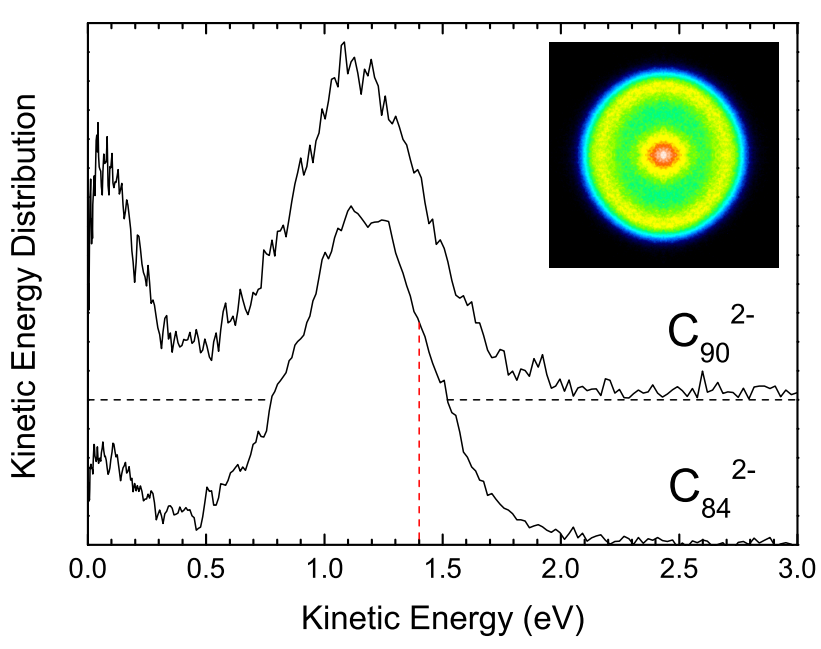

FIG. 2. (Color online) Measured KERDs of the delayed electron emission from the mass-selected fullerene dianions $\mathrm{C}_{84}{ }^{2-}$ and $\mathrm{C}_{90}{ }^{2-}$ (Inset: electron image of $\mathrm{C}_{84}{ }^{2-}$ ). The detection window is delayed by $150 \mathrm{~ns}$ with respect to the laser pulse. The red (gray) dashed line marks the RCB height $\varepsilon_{\mathrm{C}}$ and forms the limit between the tunneling and the over-the-barrier detachment. The low-energy feature (below $0.5 \mathrm{eV}$ ) results from the thermionic emission of the singly charged anions $\mathrm{C}_{84}{ }^{-}$and $\mathrm{C}_{90}{ }^{-}$, in agreement with the picture of a sequential detachment.

fullerene anions are produced from the dianions by direct detachment or by thermionic emission at a shorter time, in agreement with the picture of a sequential detachment. This picture is confirmed by a laser-heating experiment on $\mathrm{C}_{76}{ }^{2-}$ and $\mathrm{C}_{84}{ }^{2-}$ where the fractions of dianions and anions have been measured as a function of the laser fluence [14]. The high-energy feature is attributed to the emission of an electron from $\mathrm{C}_{84}{ }^{2-}$ and $\mathrm{C}_{90}{ }^{2-}$. A RCB height $\varepsilon_{\mathrm{C}}$ of $1.4 \mathrm{eV}$ has been measured for $\mathrm{C}_{84}{ }^{2-}$ by photoelectron spectroscopy in a magnetic bottle [10] and is marked by a dashed line in Fig. 2 forming the boundary between tunneling detachment $\left(\varepsilon<\varepsilon_{\mathrm{C}}\right)$ and over-the-barrier detachment $\left(\varepsilon>\varepsilon_{\mathrm{C}}\right)$. Thus the experimental KERDs demonstrate that the major part of the electrons emitted from the dianions with a delay of about 150 ns tunnel through the RCB.

The delayed electron emission of dianions $\left(\mathrm{C}_{n}{ }^{2-} \rightarrow \mathrm{C}_{n}{ }^{-}+\right.$ $\left.e^{-}\right)$is interpreted as a statistical process and is called thermionic emission by analogy with bulk matter. Its description is based on the assumption of a thermal equilibrium among all vibrational degrees of freedom. It relies on the detailedbalance principle that is based on microscopic reversibility. As both dianion and anion are assumed to be in their electronic ground state, the relevant electron binding energy $E_{b}$ is the second electron affinity $E A_{2}$ of the neutral fullerene. In the framework of the formalism introduced by Weisskopf [19], the differential rate for the emission of an electron with kinetic energy $\varepsilon$ from a dianion $\mathrm{C}_{n}{ }^{2-}$ with internal (vibrational) energy $E$ may be written as [20-22]

$$
\begin{aligned}
k(E, \varepsilon)= & \frac{m_{e}}{\pi^{2} \hbar^{3}} \frac{g_{f}}{g_{i}} \varepsilon \sigma(\varepsilon) \exp \left[-\frac{\varepsilon}{k_{B} T_{d}}\left(1+\frac{\varepsilon}{2 C_{d} T_{d}}\right)\right] \\
& \times \exp \left(-\frac{E_{b}}{k_{B} T_{e}}\right) .
\end{aligned}
$$


The microcanonical temperatures $T_{d}=T\left(E-E_{b}\right)$ and $T_{e}=T\left(E-E_{b} / 2\right)$ are, respectively, called the daughter and the emission temperature. $m_{e}$ is the electron mass; $g_{f} / g_{i}$ denotes the ratio between the electronic degeneracies in the final and the initial states $\left(g_{f}\right.$ includes the spin degeneracy of the outgoing electron). As justified below, the magnitude of $\varepsilon$ compared to $E-E_{b}$ requires introducing the second-order term of the expansion in $\varepsilon$ (this term depends on the heat capacity $C_{d}$ defined for $T=T_{d}$ ) [23]. $\sigma(\varepsilon)$ is the cross section of the reverse process, namely the attachment of an electron to the anion.

$\sigma(\varepsilon)$ is the crucial ingredient in this model. It contains all the specificities of the electron emission from MCAs (in comparison to singly charged anions or neutrals) and in particular the tunneling through the RCB. Its derivation is based on a potential curve, for which we use the classical expression of the energy of a point charge $-e$ at a distance $r$ from the center of a singly negatively charged dielectric sphere. Following Ref. [14], in addition to the Coulomb and $1 / r^{4}$ terms of the expansion, we include the $1 / r^{6}$ term to account for a finite-size sphere instead of a point polarizability and we derive an effective sphere radius $r_{0}$ from the polarizability $\alpha$ and the dielectric constant $\varepsilon_{r}$ using $\alpha=r_{0}^{3}\left(\varepsilon_{r}-1\right) /\left(\varepsilon_{r}+2\right)$. For the dielectric constant, we use a value of $\varepsilon_{r}=3.6$ as recommended for $C_{60}$ [24]. Let us notice that with this value of dielectric constant, a classical electrostatic model leads to $E A_{2}$ values in agreement with the experiment [14]. Finally the potential may be written [14]

$$
V(r)=\frac{e^{2}}{r}-\frac{\alpha}{2 r^{4}}\left[1+\frac{2 \varepsilon_{r}+4}{2 \varepsilon_{r}+3}\left(\frac{r_{0}}{r}\right)^{2}\right] .
$$

The polarizabilities of $C_{84}\left(\alpha=130 \AA^{3}\right)$ and $C_{90}(\alpha=$ $\left.145 \AA^{3}\right)$ have been extrapolated from the measurements for $\mathrm{C}_{60}$ and $\mathrm{C}_{70}[25,26]$ using a $n^{3 / 2}$ law in agreement with the molecular cage structure. The potential curve $V(r)$ for $\mathrm{C}_{84}{ }^{2-}$ is displayed in Fig. 1. For $n=84$, the RCB maximum reached at $R_{\mathrm{C}}=8.1 \AA$ is given by $\varepsilon_{\mathrm{C}}=1.40 \mathrm{eV}\left(R_{\mathrm{C}}=8.4 \AA\right.$ and $\varepsilon_{\mathrm{C}}=1.35 \mathrm{eV}$ for $n=90$ ). Thanks to the introduction of the $1 / r^{6}$ term in the potential $V(r)$, the $\varepsilon_{\mathrm{C}}$ value of $\mathrm{C}_{84}$ is in agreement with the low-energy cutoff of photoelectron spectra measured with a magnetic bottle [10]. Besides, the second electron affinity $E A_{2}$ of $\mathrm{C}_{84}$ and $\mathrm{C}_{90}$ amounts, respectively, to 0.75 and $0.84 \mathrm{eV}[10,14]$.

For the attachment cross section, we use a hard-sphere cross section with a capture radius equal to the distance $R_{C}$ at which the interaction potential reaches its maximum value $\varepsilon_{\mathrm{C}}$. Below the barrier, this expression is weighted by the electron tunneling probability $P(\varepsilon)$. Finally the attachment cross section reads

$$
\begin{aligned}
& \sigma(\varepsilon)=P(\varepsilon) \pi R_{\mathrm{C}}^{2} \text { for } \varepsilon<\varepsilon_{\mathrm{C}}, \\
& \sigma(\varepsilon)=\pi R_{\mathrm{C}}^{2} \text { for } \varepsilon>\varepsilon_{\mathrm{C}} .
\end{aligned}
$$

The Wentzel-Kramers-Brillouin (WKB) expression for one-dimensional tunneling [27] has been used for the numerical calculation of the probability $P(\varepsilon)$,

$$
P(\varepsilon)=\exp \left\{-\frac{2}{\hbar} \int \sqrt{2 m_{e}[V(r)-\varepsilon]} d r\right\} .
$$

In an experimental study of the direct electron emission of $\mathrm{PtBr}_{4}^{2-}$, the evolution of the tunneling probability as a function of the kinetic energy was well reproduced by the WKB expression [28]. It must be underlined also that tunneling was disregarded in the previous applications of the detailed balance to statistical electron emission from MCAs $[12,14]$.

The KERD of the electrons emitted from the dianions of a given internal energy $E$ is deduced from the differential rate for electron emission [Eq. (1)] [22],

$$
f(\varepsilon) \propto \varepsilon \sigma(\varepsilon) \exp \left[-\frac{\varepsilon}{k_{B} T_{d}}\left(1+\frac{\varepsilon}{2 C_{d} T_{d}}\right)\right] .
$$

In order to compare this distribution with the experimental one, the daughter temperature $T_{d}$ must be determined. This is done using the integrated decay rate $K(E)$ for the emission of an electron from a dianion of internal energy $E$. The decay rate $K(E)$ shows strong variations with the internal energy $E$, which implies a narrow internal energy distribution for the dianions decaying during the experimental time window. This distribution may be reduced to a single value $\bar{E}$ defined such that

$$
K(\bar{E})=\frac{1}{\tau},
$$

with $\tau$ the delay corresponding to the middle of the experimental time window [29]. In the present work, $\tau=150 \mathrm{~ns}$. Equation (6) relies on the assumption that the electron emission is the only decay channel of the fullerene dianions, which has been shown by the measurement of ion mass spectra in a Penning trap after laser irradiation [14]. For the numerical resolution of Eq. (6), a caloric curve is used [17].

The ratio $g_{f} / g_{i}$ of $\mathrm{C}_{84}$ amounts to 3.11, accounting for its two isomers [14]. Such an estimate cannot be provided for $\mathrm{C}_{90}$ as no information is available on the electronic degeneracy of its five isomeric forms [30]. By numerically solving Eq. (6) for $\mathrm{C}_{84}{ }^{2-}$, we find an internal energy $\bar{E}=14.5 \mathrm{eV}$ corresponding to a daughter temperature $T_{d}=1175 \mathrm{~K}$. When comparing the average kinetic energy (which amounts to $1.15 \mathrm{eV}$ ) to $\bar{E}-E_{b}$, it appears justified to account for the second-order term in the expansion in $\varepsilon$ of the vibrational density of states. Besides, the large amount of vibrational energy $\bar{E}$ before thermionic emission supports a multiphoton absorption process: The $\mathrm{C}_{84}{ }^{2-}$ dianions decaying during the experimental time window have been expected to absorb six photons (at the laser wavelength of $532 \mathrm{~nm}$ ).

Figure 3 shows experimental and theoretical KERDs of delayed electron emission from $\mathrm{C}_{84}{ }^{2-}$ and $\mathrm{C}_{90}{ }^{2-}$. The contribution of the thermionic emission of the singly charged anions has been subtracted from the experimental KERDs of Fig. 2 using the spectra of Ref. [17]. The theoretical KERDs have been convoluted by the instrumental resolution. For $\mathrm{C}_{84}{ }^{2-}$, the KERD parametrized by the temperature calculated with the detailed-balance model $\left(T_{d}=1175 \mathrm{~K}\right)$ is in very good agreement with the experiment. We emphasize that no adjustable parameter has been used for this comparison (except an intensity factor). In order to complement the comparison between experiment and theory, the experimental KERD of $\mathrm{C}_{90}{ }^{2-}$ has been fitted with the theoretical expression given by Eq. (5) using $T_{d}$ as a free parameter (on top of the intensity factor). A temperature $T_{d}=1210 \mathrm{~K}$ is deduced. An 


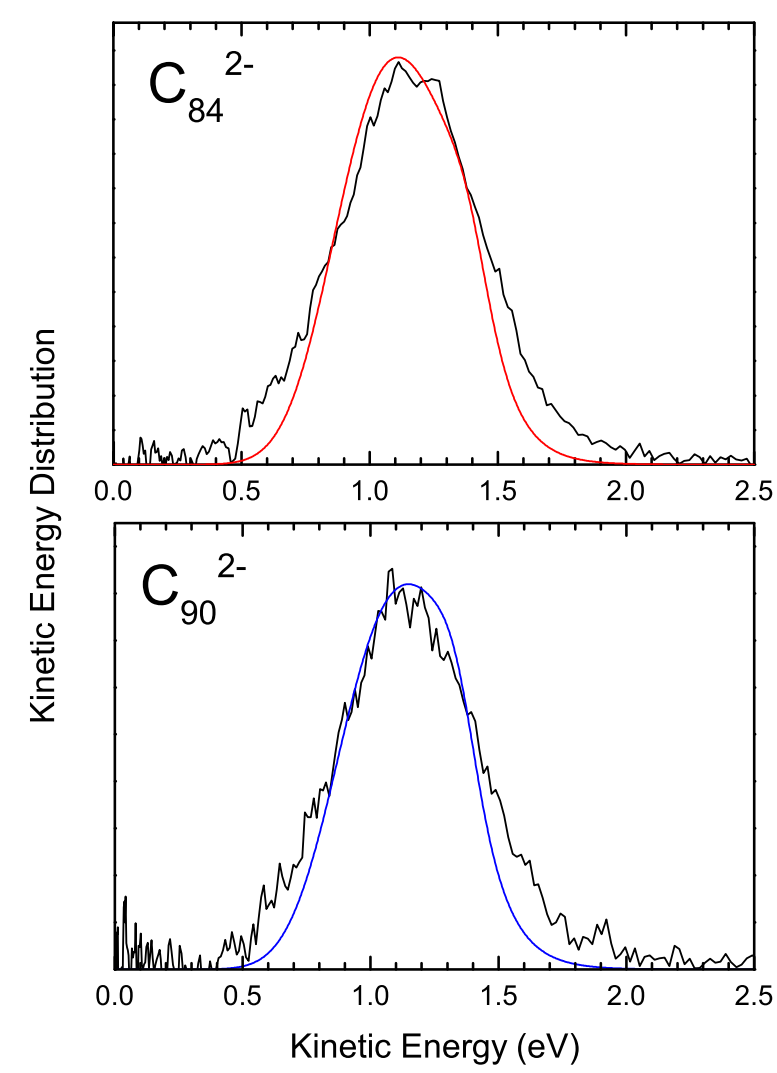

FIG. 3. (Color online) Comparison between measured and calculated KERDs of delayed electron emission from $\mathrm{C}_{84}{ }^{2-}$ and $\mathrm{C}_{90}{ }^{2-}$. The measured KERDs are displayed as black lines. For $\mathrm{C}_{84}{ }^{2-}$ (top), the theoretical KERD [red (gray) line] is calculated without adjustable parameter in the framework of the detailed-balance model. For $\mathrm{C}_{90}{ }^{2-}$ (bottom), the blue (gray) curve is a fit to the experimental KERD using the detailed-balance expression and considering $T_{d}$ as a free parameter. Both theoretical KERDs are convoluted by the instrumental resolution.

estimate is available for all the model parameters except the ratio $g_{f} / g_{i}$ of the electronic degeneracies which consequently can be deduced from the temperature. A value of 2.64 is found, which is plausible and close to the 3.11 value for $\mathrm{C}_{84}{ }^{2-}$. For both dianions, the shape of the experimental curve is well but not fully reproduced by the theory. Most likely the agreement would be even better if the description of the reverse process was improved by going beyond the hard-sphere approximation or the WKB theory. Nevertheless even with a simple description of the attachment, the agreement with the experiment is already satisfactory and strongly supports the statistical nature of the delayed electron emission.

Let us briefly discuss the ongoing processes. During the 23-ns laser pulse, the fullerene dianions sequentially absorb photons, the excitation energy being rapidly transferred from the electronic to the vibrational degrees of freedom via intramolecular conversion. The resulting dianions are vibrationally hot but remain in their electronic ground state. They statistically decay by electron emission after a delay directly related to their vibrational temperature (hotter dianions decay faster on the average). During this process, part of the vibrational energy is transferred to a single electron. This energy is shared between the second electron affinity and the kinetic energy. The nonconservation of the vibrational energy is a feature of thermionic emission and an important difference with the adiabatic process proposed for fluorescein [4]. Even if a systematic time-resolved study of delayed electron emission is not possible under our experimental conditions, it is interesting to discuss, at least qualitatively, the changes of the KERD when the delay varies. If the delay increases, the temperature of the decaying dianions and the average kinetic energy decrease. At some point, the over-the-barrier detachment should even disappear and all the ejected electrons should tunnel through the RCB.

In summary, the KERD of delayed electron emission from fullerene dianions has been measured and analyzed in the framework of a detailed-balance model which includes the electron tunneling through the RCB. The very good agreement between experiment and theory provides quantitative evidence for a thermal tunneling detachment. The detailed-balance formalism and the microcanonical temperature are reinforced as a suitable framework for the description of statistical processes in molecular systems. Thermionic emission appears as a probe of the RCB complementary to the direct photodetachment.

Financial support of the French "Agence Nationale de la Recherche" (ANR) through Project No. ANR-06-BLAN-0041 is acknowledged.
[1] X.-B. Wang and L.-S. Wang, Nature 400, 245 (1999).

[2] X.-P. Xing, X.-B. Wang, and L.-S. Wang, Phys. Rev. Lett. 101, 083003 (2008).

[3] X.-B. Wang and L.-S. Wang, Annu. Rev. Phys. Chem. 60, 105 (2009).

[4] D. A. Horke, A. S. Chatterley, and J. R. R. Verlet, Phys. Rev. Lett. 108, 083003 (2012).

[5] P. D. Dau, H.-T. Liu, J.-P. Yang, M.-O. Winghart, T. J. A. Wolf, A.-N. Unterreiner, P. Weis, Y.-R. Miao, C.-G. Ning, M. M. Kappes, and L.-S. Wang, Phys. Rev. A 85, 064503 (2012).

[6] J. R. R. Verlet, D. A. Horke, and A. S. Chatterley, Phys. Chem. Chem. Phys. 16, 15043 (2014).
[7] O. T. Ehrler, J. M. Weber, F. Furche, and M. M. Kappes, Phys. Rev. Lett. 91, 113006 (2003).

[8] O. T. Ehrler, F. Furche, J. M. Weber, and M. M. Kappes, J. Chem. Phys. 122, 094321 (2005).

[9] X.-B. Wang, H.-K. Woo, X. Huang, M. M. Kappes, and L.-S. Wang, Phys. Rev. Lett. 96, 143002 (2006).

[10] X.-B. Wang, H.-K. Woo, J. Yang, M. M. Kappes, and L.-S. Wang, J. Phys. Chem. C 111, 17684 (2007).

[11] C. Cauchy, J. M. Bakker, Y. Huismans, A. Rouzée, B. Redlich, A. F. G. van der Meer, C. Bordas, M. J. J. Vrakking, and F. Lépine, Phys. Rev. Lett. 110, 193401 (2013).

[12] R. N. Compton, A. A. Tuinman, C. E. Klots, M. R. Pederson, and D. D. Patton, Phys. Rev. Lett. 78, 4367 (1997). 
[13] B. Concina, M. Neumaier, O. Hampe, and M. M. Kappes, Int. J. Mass Spectrom. 252, 110 (2006).

[14] B. Concina, M. Neumaier, O. Hampe, and M. M. Kappes, J. Chem. Phys. 128, 134306 (2008).

[15] D. A. Horke, A. S. Chatterley, and J. R. R. Verlet, J. Chem. Phys. 139, 084302 (2013).

[16] A. S. Chatterley, D. A. Horke, and J. R. R. Verlet, Phys. Chem. Chem. Phys. 16, 489 (2014).

[17] B. Concina, F. Lépine, and C. Bordas, Phys. Rev. A 90, 033415 (2014).

[18] C. Bordas, F. Paulig, H. Helm, and D. L. Huestis, Rev. Sci. Instrum. 67, 2257 (1996).

[19] V. Weisskopf, Phys. Rev. 52, 295 (1937).

[20] J. U. Andersen, E. Bonderup, and K. Hansen, J. Chem. Phys. 114, 6518 (2001).

[21] J. U. Andersen, E. Bonderup, and K. Hansen, J. Phys. B 35, R1 (2002).

[22] F. Lépine and C. Bordas, Phys. Rev. A 69, 053201 (2004).
[23] F. Calvo, P. Parneix, and F. X. Gadéa, J. Phys. Chem. A 110, 1561 (2006).

[24] C. R. Snyder and J. F. Douglas, J. Phys. Chem. B 104, 11058 (2000).

[25] R. Antoine, P. Dugourd, D. Rayane, E. Benichou, M. Broyer, F. Chandezon, and C. Guet, J. Chem. Phys. 110, 9771 (1999)

[26] I. Compagnon, R. Antoine, M. Broyer, P. Dugourd, J. Lermé, and D. Rayane, Phys. Rev. A 64, 025201 (2001).

[27] L. D. Landau and E. M. Lifshitz, Quantum Mechanics: NonRelativistic Theory (Butterworth-Heinemann, Oxford, Boston, 1991).

[28] D. Löffler, J. M. Weber, and M. M. Kappes, J. Chem. Phys. 123, 224308 (2005).

[29] B. Concina, B. Baguenard, F. Calvo, and C. Bordas, J. Chem. Phys. 132, 104307 (2010).

[30] Y. Achiba, K. Kikuchi, Y. Aihara, T. Wakabayashi, Y. Miyake, and M. Kainosho, Mater. Res. Soc. Symp. Proc. 359, 3 (1995). 